\title{
3D Modeling of the Mechanical Behavior of Ceramics with Pores of Different Size
}

\author{
Alexey Yu. Smolin ${ }^{1,2, \text { a) }}$, Galina M. Eremina ${ }^{2, \text { b) }}$, Igor S. Konovalenko, ${ }^{1, \text { c) }}$ \\ and Sergey G. Psakhie ${ }^{3,4, d)}$ \\ ${ }^{1}$ Institute of Strength Physics and Materials Science SB RAS, Tomsk, 634055, Russia \\ ${ }^{2}$ National Research Tomsk State University, Tomsk, 634050, Russia \\ ${ }^{3}$ Institute of High Technology Physics, National Research Tomsk Polytechnic University, Tomsk, 634050, Russia \\ ${ }^{4}$ Skolkovo Institute of Science and Technology, Skolkovo, 143025, Russia

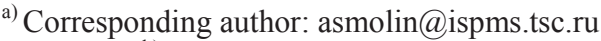 \\ b) anikeeva@ispms.tsc.ru \\ c)igkon@ispms.tsc.ru \\ d) sp@ispms.tsc.ru
}

\begin{abstract}
Movable cellular automaton method was used for simulating uniaxial compression of 3D porous ceramic samples. Pores were considered explicitly by removing randomly selected automata from the original FCC packing. Distribution of pores in space, their size and the total fraction were varied. It is shown that the relation between mechanical properties of the material and its porosity significantly depends on the pore size. Thus, value of the elastic modulus of the samples with large pores is greater than that of the samples with small pores by average value of $3 \%-16 \%$. Strength value of the samples with large pores is less than that of the samples with small pores by average value of $12 \%$ up to the porosity of 0.55 , and then becomes to be greater. When the samples contain small and large pores there is a maximum of mechanical properties at ratio of volumes of large and small pores of about 0.75 .
\end{abstract}

Keywords: porous ceramics, mechanical properties, modeling, movable cellular automata

\section{INTRODUCTION}

Porous materials are widely used in modern industry and have a great potential for using in future. It is well known that physical and mechanical properties of such materials depend both on volume and structure of pores. For example, the dependence of strength and elastic properties of porous materials on porosity is determined by the pore morphology (this problem has a long history [1] and is very interesting up till now [2-6]). The modern methods of calculating the effective elastic properties of composites, such as method of equivalent field and differential selfconsistent method, allow taking into account the pore morphology and are considered to be enough exact for small and high concentration of pores. But these methods are based on the assumption that material matrix is a continuum, and hence do not allow to take into account its microstructure. That is why the direct modeling of porous materials under mechanical loading is of high interest nowadays. Computational methods of continuum $[2,3]$ and particle mechanics are used for this purpose [5-8]. Note that the methods based on particle approach allow correct modeling of deformation and have their advantages for modeling the fracture.

The purpose of this study was to reveal the influence of pore size on the dependence of the strength and elastic properties of porous ceramics on its porosity using a particle-based computational model [8]. This model is based on the method of movable cellular automata used herein for modeling porous ceramics samples in uniaxial compression.

International Conference on Physical Mesomechanics of Multilevel Systems 2014

AIP Conf. Proc. 1623, 591-594 (2014); doi: 10.1063/1.4899014

(C) 2014 AIP Publishing LLC 978-0-7354-1260-6/\$30.00 


\section{MODEL DESCRIPTION}

The movable cellular automata is a new efficient numerical method in particle mechanics that is different from methods in the traditional continuum mechanics [8-10]. Within the frame of movable cellular automata, it is assumed that any material is composed by a certain amount of elementary objects (automata), which interact among each other and can move from one place to another, thereby simulating a real deformation process. The automaton motion is governed by the Newton-Euler equations.

The forces acting on automata are calculated using the deformation parameters, i.e. relative overlap, tangential displacement and rotation, and conventional elastic constants, i.e. shear and bulk moduli. A distinguishing feature of the method is calculating of forces acting on the automata within the framework of multi-particle interaction [8-10], which provides for an isotropic behavior of the simulated medium. Moreover, stress tensor components could be calculated for the automaton taking into account all the forces acting on it $[9,10]$, which enables the realization of various models of plasticity behavior developed in the frame of continuum mechanics.

A pair of elements might be considered as a virtual bistable cellular automaton (bound and unbound states), which permits the simulation of fracture by the movable cellular automata. Switching of a pair of automata to an unbound state would result in a changeover in the forces acting on the elements; in particular, they would not resist moving away from one another.

Herein the movable cellular automata method was used for 3D computer simulation of mechanical behavior of a porous material under uniaxial compression. Response function of automata used in this study corresponded to $\mathrm{ZrO}_{2}\left(\mathrm{Y}_{2} \mathrm{O}_{3}\right)$ ceramics with average size of pores commensurable with the grain size of the material and porosity equal to 0.02 (i.e. Young's modulus of intact material was equal to $80 \mathrm{GPa}$ and Poisson's ratio was 0.3). According to the pore distribution diagram of this material [11] the automaton size in the computations was equal to $1 \mu \mathrm{m}$.

All the modeled samples were bricks with a square base; the base side $a=60 \mu \mathrm{m}$ and the height of the brick $h=$ $2 a$. To simulate loading, one and the same velocity in the vertical direction was assigned for all the automata of the upper layer, while the automata of the lower layer were fixed in the vertical direction [8]. Effective mechanical properties of a model sample were determined from the calculated loading diagram: the elastic modulus from the slope of the first linear part of the diagram and the strength from its maximum.

Small pores were generated by removing single automata selected randomly from the initial FCC structure. A large pore was generated by removing single automaton and all its neighbors. The porosity values were varied from 0 up to 0.7 .

\section{MODELING RESULTS}

Let us consider the dependence of the calculated effective elastic modulus of 3D samples on its porosity. In Fig. 1 each point represents the value averaged on five representative samples with various pore distributions in space. As shown in [8], this plot can be divided into two characteristic parts connected with porous structure: the first corresponds to closed pores $(0-0.25)$, the second corresponds to interconnected pores $(0.25-0.65)$. The points corresponding to closed pores best fit the following equation

$$
E=E_{0}\left(1-C / C_{\max }\right)^{m},
$$

whereas the points corresponding to interconnected pores fit much better another equation

$$
E=E_{0}\left(C_{0}-C / C_{\max }\right)^{m}
$$

where $C_{0}, C_{\max }$ and $m$ are adjustable parameters. The physical meaning of these parameters is clear. $C_{\max }$ means the maximal value of porosity of a sample that have any strength for the given pore morphology. For example, the procedure used for generating porosity in this work has a limit beyond which the remaining automata would not interact with each other. They would "hang" in the air and not resist to any load. Indeed, it is well known that real materials having large porosity have pore wall thickness much less than the pore size. Thus, to generate larger porosity for samples based on FCC packing of automata it is necessary to remove not a single automata, but also all its neighbors. Parameter $C_{0}$ in Eq. (2) means that at zero porosity the modulus is not equal to the value of intact material, but it allows adjusting to exact value at other porosity value, namely at percolation limit. 


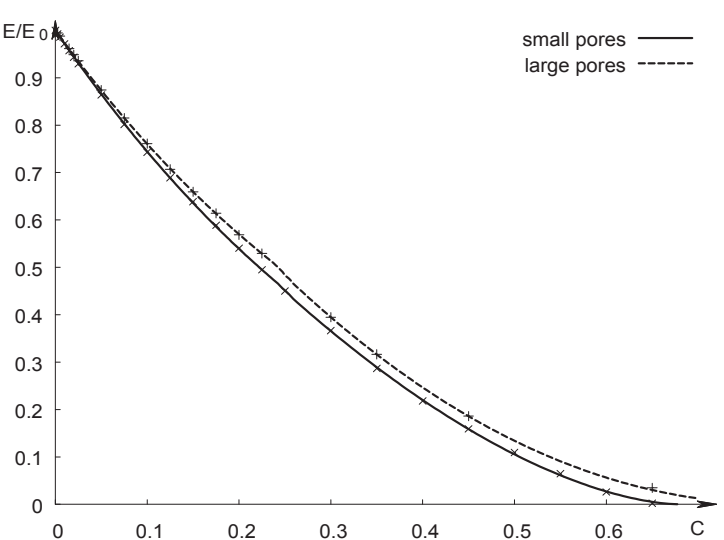

FIGURE 1. Plots of normalized elastic modulus of modeled ceramics versus its porosity

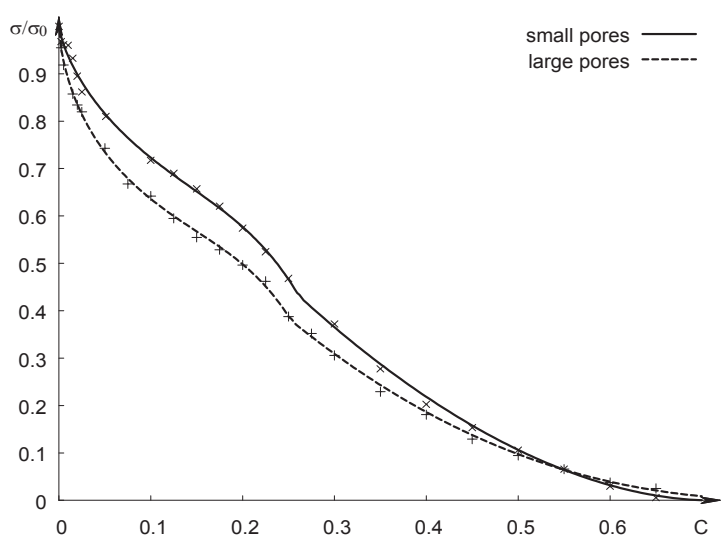

FIGURE 2. Plots of normalized strength of modeled ceramics versus its porosity

Data in Fig. 1 shows that the samples with large pores have greater elastic modulus than that with small pores and relative difference of the modulus increases with increasing porosity.

Now, let us consider the dependence of the effective strength of 3D samples on its porosity. In Fig. 2 again each point represents the value averaged on five representative samples with various pore distributions in space. Note that the calculation results for the samples with closed porosity in Fig. 2 best fit the following equation

$$
\sigma=\sigma_{0} \frac{1-\left(C / C_{\max }\right)^{m}}{1+\left(C / C_{n}\right)^{n}},
$$

where $C_{\mathrm{n}}, C_{\max }, n$ and $m$ are adjustable parameters [8]. Whereas the modeling results for strength of the samples with larger porosity $(0.25-0.65)$ fit very well the Eq. (2), i.e. the same equation as for the dependence of elastic modulus on porosity, but with different values of adjustable parameters [8].

From the data presented in Fig. 2 one can conclude that a) percolation transition manifests itself much better on strength dependence on porosity; and b) as opposed to elastic modulus the strength value of the samples with large pores is less than that of the samples with small pores by average value of $12 \%$ up to the porosity of 0.55 and then becomes to be greater.

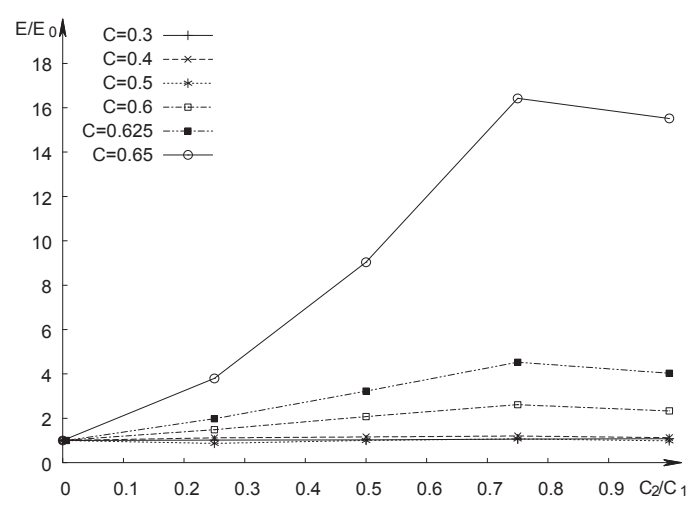

(a)

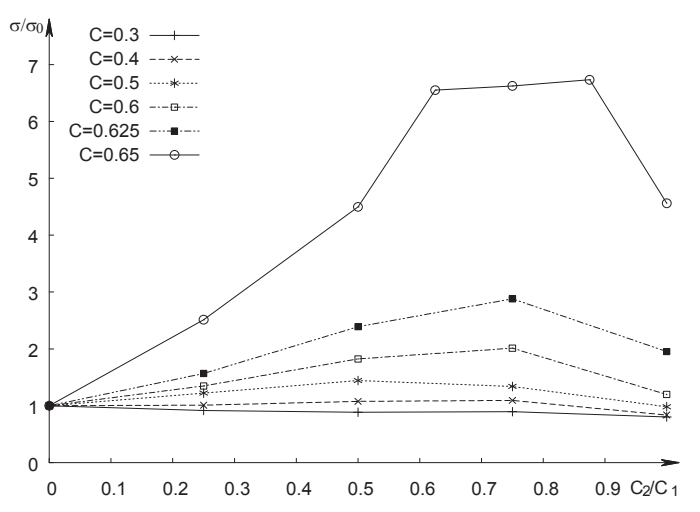

(b)

FIGURE 3. Plots of normalized elastic modulus (a) and strength (b) of modeled ceramics versus ratio of volume fractions of large $C_{2}$ and small $C_{1}$ pores for various total porosity $C$ 
Further we studied the samples with various ratios of small and large pores. Plots of normalized mechanical properties of the modeled ceramics versus ratio of fractions of different pores are shown in Fig. 3. Here the most interesting is the maxima in plots at ratio of volumes of large and small pores of about 0.75 . This could be explained by the fact that when all pores are large and we try to add several small pores instead of one large pore then it is possible to get thicker pore walls. Again, these maxima show themselves much better in plots for strength and for larger porosity.

\section{SUMMARY}

Deformation and fracture of porous ceramics have been successfully simulated using the method of movable cellular automata in wide range of porosity. To reveal the influence of pore size on the porosity dependence of mechanical properties of the material we explicitly consider equiaxed pores of different size stochastically distributed in space. The main results are summarized as follows. Elastic modulus of the samples with large pores is greater than that of the samples with small pores. Strength of the samples with large pores is less than that of the samples with small pores up to the porosity of 0.55 , and then becomes to be greater. When the samples contain small and large pores there is a maximum of mechanical properties at ratio of volumes of large and small pores of about 0.75 .

\section{ACKNOWLEDGMENTS}

The investigation has been carried out at partial financial support of the Program for Basic Research of State Academies of Sciences for 2013-2016 (project No. III.23.2.3).

\section{REFERENCES}

1. T. T. Wu, Int. J. Sol. Struct. 2, 1 (1966).

2. V. A. Skripnyak, E. G. Skripnyak, A. A. Kozulin, V. V. Skripnyak, and M. V. Korobenkov, Russ. Phys. J. 52(12), 1300 (2009).

3. Yu. A. Kostandov, P. V. Makarov, M. O. Eremin, I. Yu. Smolin, and I. E. Shipovskii, Int. J. Appl. Mech. 49(1), 95 (2013).

4. S. N. Kulkov, S. P. Buyakova, A. Yu. Smolin, N. V. Roman, and S. A. Kinelovskii, Tech. Phys. Lett. 37(4), 360-3 (2011).

5. M. P. J. Schöpfer, S. Abe, C. Childs, and J. J. Walsh, Int. J. Rock Mech. Mining Sci. 46, 250 (2009).

6. Ig. S. Konovalenko, A. Yu. Smolin, S. Yu. Korostelev, and S. G. Psakh'e, Tech. Phys. 54(5), 758 (2009).

7. A. F. Revuzhenko and S. V. Klishin, Phys. Mesomech. 16(2), 152 (2013).

8. A. Yu. Smolin, N. V. Roman, I. S. Konovalenko, G. M. Eremina, S. P. Buyakova, and S. G. Psakhie, Eng. Fract. Mech., DOI: 10.1016/j.engfracmech.2014.04.001 (in press).

9. S. Psakhie, E. Shilko, A. Smolin, S. Astafurov, and V. Ovcharenko, Fract. Int. Struct. 24, 26 (2013).

10. S. G. Psakhie, E. V. Shilko, A. Yu. Smolin, A. V. Dimaki, A. I. Dmitriev, Ig. S. Konovalenko, S. V. Astafurov, and S. Zavshek, Phys. Mesomech. 14(5-6), 224 (2011).

11. S. N. Kulkov, Phys. Mesomech. 11(1-2), 29 (2008). 